\section{Are MELAS and diabetes mellitus caused solely by the same mutation at np 3243 of the mitochondrial gene?}

\section{Dear Sir,}

Kishimoto et al. [1] reported on six patients with diabetes mellitus carrying an A-to-G mutation at nucleotide position (np) 3243 in the mitochondrial (mt) tRNA ${ }^{\mathrm{LEU}(\mathrm{UUR})}$ gene, who had phenotypes distinct from those commonly observed in diabetes. Previous reports including theirs, however, failed to make clear why this mutation is associated with two clinically distinct diseases: diabetes mellitus and MELAS (mitochondrial encephalomyopathy, lactic acidosis, and stroke-like episodes). This mutation was originally identified in MELAS, one of several mitochondrial genetic diseases that include the KearnsSayre/chronic progressive ophthalmoplegia complex, MERRF (myoclonic epilepsy with ragged red fibers), LHON (Leber's hereditary optic neuropathy) and NARP (neuropathy, ataxia, and retinitis pigmentosum)/maternally inherited Leigh syndrome. It was a great surprise for us when the same mutation at np 3243 was described as associating with diabetes mellitus [2]. This mutation exists in about $1 \%$ of randomly selected non-insulin-dependent diabetic (NIDDM) patients [3], and is rarely seen in autoimmune type 1 (insulin-dependent) diabetes (IDDM) [4]. It was also surprising that the single mutation at $\mathbf{n p} 3243$ is associated with as many as $1 \%$ of NIDDM patients, because this prevalence is quite high compared with previously reported gene mutations in insulin, insulin receptor [5] or glucokinase genes. But little is known concerning what really defines the clinical characteristics of the two distinct diseases. It has been believed that clinical varieties in patients with mtDNA mutations are attributable to the different populations of mutant mtDNA coexisting with wildtype DNA (heteroplasmy) in the mitochondrion and to the differing sensitivity of tissues and organs to respiratory chain dysfunction. But some reports deny the presence of an increased proportion of mutant DNA in symptomatic organs [6]. We thought that some factor other than the 3243 base pair (bp) mutation might contribute to the aetiology of the different clinical phenotypes found in these patients, and analysed three families from our hospital with the 3243 bp mutation [3] along with families reported in recent literature [1, 7] (Table 1). The families were classified into two groups by the associated diseases of the proband; MELAS families in which the proband was associated with MELAS, and diabetic families in which the proband does not have MELAS but has diabetes (Table 1). Of the $28 \mathrm{MELAS}$ families, we found members with diabetes or impaired glucose tolerance (IGT) in 22-27 (no description of diabetes in five families). The total number of patients with MELAS was 55 (2.0 subjects per family), and for those with diabetes it was 72 (3.1 per family). In the 33 diabetic families, 118 patients had diabetes ( 3.6 per family) (Table 1), but no MELAS family members were identified. The prevalence of diabetes was similar between the two groups, but the prevalence of MELAS was quite different. The ratio of patients with MELAS to patients with diabetes was 0.76 in MELAS families and 0 in diabetic families, indicating an increased prevalence of this rare disease in MELAS families. Many members with MELAS, excluding children, frequently had diabetes and their family members were also frequently diabetic, while none of the diabetic family members had ME-

Corresponding author: Dr. M. Odawara, Institute of Clinical Medicine, The University of Tsukuba, 1-1-1, Tennodai, Tsukuba-city, Ibaraki, 305, Japan
Table 1. Analysis of families with 3243 bp mutation

\begin{tabular}{lcccc}
\hline & \multicolumn{2}{l}{ MELAS Diabetic or IGT } & $\begin{array}{l}\text { Total } \\
\text { families }\end{array}$ & References \\
\cline { 2 - 4 } & Family & & 11 & 7 \\
MELAS & $11(11)$ & $11(26)$ & 7 & 11 \\
families & $7(7)$ & $6(6)$ & 5 & 8 \\
& $5(17)$ & ND & 2 & 12 \\
& $2(16)$ & $2(16)$ & 3 & $3,13,14$ \\
& $3(4)$ & $3(13)$ & 28 & \\
Diabetic & $28(55)$ & $22-27(72)$ & 11 & 7 \\
families & 0 & $11(29)$ & & \\
& 0 & $6(16)$ & 6 & 1 \\
& 0 & $5(13)$ & 5 & 15 \\
& 0 & $4(18)$ & 4 & 16 \\
& 0 & $7(42)$ & 7 & $2,3,17,18,19$ \\
& 0 & $33(118)$ & 33 & \\
\hline
\end{tabular}

Numbers in parentheses indicate numbers of subjects

LAS. In five families reported by Ciafaloni et al. [8], 17 had MELAS (3.4 MELAS patients per family) while in two families, five or six patients were affected, implying increased susceptibility to MELAS in these families. Though we cannot totally exclude the possibility that a high prevalence of diabetes may be responsible for the increased prevalence of diabetes in diabetic families, the presence of this rare disease in many members from MELAS families suggests that they have a common susceptibility factor that diabetic family members lack. Recently, patients with other mutations causing amino acid substitutions along with the mutations in the tRNAs of the mtDNA are reported to have more severe clinical phenotypes and shorter life-spans than those with one of the two kinds of mutations [9]. In another report, the dizygotic brother of a patient with an 'incomplete' form of MELAS with the $3243 \mathrm{bp}$ mutation, was found not to carry this mutation despite having associated myopathy and a subclinical leukoencephalopathy [10]. These observations also suggest that the 3243 bp mutation may not be the only factor that defines the clinical phenotypes of MELAS patients carrying the $3243 \mathrm{bp}$ mutation. This is important when patients are informed about the mutation they are carrying, because some of these patients feel anxious about the possibility of developing MELAS, or it may affect their feelings about having children. In conclusion, though the A-to-G mutation at $\mathrm{np} 3243$ is associated with both diabetes and MELAS, our observations suggest the possibility that factor(s) other than this mutation is (are) involved in the pathogenesis of MELAS.

Yours sincerely,

M. Odawara, K. Yamashita

\section{References}

1. Kishimoto M, Hashiramoto M, Araki S et al. (1995) Diabetes mellitus carrying a mutation in the mitochondrial

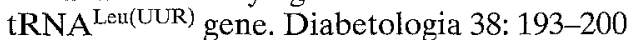

2. Van den Ouweland JMW, Lemkes HHPJ, Ruitenbeek W et al. (1992) Mutation in mitochondrial tRNA ${ }^{\text {Leu(UUR) }}$ gene in a large pedigree with maternally transmitted type II diabetes mellitus and deafness. Nature Genet 1: 368-371

3. Odawara M, Sasaki K, Yamashita K (1995) Prevalence and clinical characterization of Japanese diabetes mellitus with an A-to-G mutation at nucleotide 3243 of the mitochondrial tRNA ${ }^{\text {Leu(UUR) }}$ gene. J Clin Endocrinol Metab 80: 1290-1294 
4. Odawara M, Sasaki K, Nagafuchi S, Tanae A, Yamashita K (1994) Lack of association between mitochondrial gene mutation np 3243 and type 1 diabetes mellitus and autoimmune thyroid diseases. Lancet 344: 1086

5. Odawara M, Kadowaki T, Yamamoto-Honda R et al. (1989) Human diabetes associated with a mutation in the tyrosine kinase domain of the insulin receptor. Science 245: 66-68

6. Love S, Nicoll JAR, Kinrade E (1993) Sequencing and quantitative assessment of mutant and wild-type mitochondrial DNA in paraffin sections from cases of MELAS. J Pathol 170: 9-14

7. Kadowaki T, Kadowaki H, Mori Yet al (1994) A subtype of diabetes mellitus associated with a mutation of mitochondrial DNA. N Engl J Med 330: 962-968

8. Ciafaloni E, Ricci E, Shanske S et al. (1992) MELAS: clinical features, biochemistry, and molecular genetics. Ann Neurol 31: 391-398

9. Ozawa T, Katsumata K, Hayakawa M et al. (1995) Mitochondrial DNA mutations and survival rate. Lancet 345: 189

10. Degoul F, Diry M, Pou-Serradell A, Lloreta J, Marsac C (1994) Myo-leukoencephalopathy in twins: study of 3243myopathy, encephalopathy, lactic acidosis, and stroke-like episodes mitochondrial DNA mutation. Ann Neurol 35: 365-370

11. Suzuki S, Hinokio Y, Hirai S, et al. (1994) Pancreatic betacell secretory defect associated with mitochondrial point mutation of the tRNA ${ }^{\text {LEU(UUR) }}$ gene: a study in seven families with mitochondrial encephalomyopathy, lactic acidosis and stroke-like episodes (MELAS). Diabetologia 37: 818825
12. Sue CM, Holmes-Walker DJ, Morris JGL, et al. (1993) Mitochondrial gene mutations and diabetes mellitus. Lancet 341: 437-438

13. Schulz JB, Klockgether T, Kichgans J, et al. (1993) Mitochondrial gene mutations and diabetes mellitus. Lancet 341: 438-439

14. Remes AM, Majamaa K, Herva R, et al. (1993) Adult-onset diabetes mellitus and neurosensory hearing loss in maternal relatives of MELAS patients in a family with the tRNA $^{\text {Leu(UUR) }}$ mutation. Neurology 43: 1015-1020

15. Otabe S, Sakura H, Shimokawa K, et al. (1994) The high prevalence of diabetic patients with a mutation in the mitochondrial gene in Japan. J Clin Endocrinol Metab 79: 768771

16. Katagiri H, Asano T, Ishihara H, et al. (1994) Mitochondrial diabetes mellitus: prevalence and clinical characterization of diabetes due to mitochondrial tRNA ${ }^{\text {Leu(UUR) }}$ gene mutation in Japanese patients. Diabetologia 37: 504510

17. van den Ouweland MJW, Lemkes HHPJ, Trembath RC, et al. (1994) Maternally inherited diabetes and deafness is a distinct subtype of diabetes and associates with a single point mutation in the mitochondrial tRNA ${ }^{\text {Leu(UUR) }}$ gene. Diabetes 43: 746-751

18. Alcolado JC, Majid A, Brockington M, et al. (1994) Mitochondrial gene defects in patients with NIDDM. Diabetologia 37: 372-376

19. Reardon W, Ross RJM, Sweeney MG, et al. (1992) Diabetes mellitus associated with a pathogenic point mutation in mitochondrial DNA. Lancet 340: 1376-1379

\section{Response from the authors}

\section{Dear Sir,}

Drs. Odawara and Yamashita are to be thanked for their valuable comments. They raised the important point in their letter that a certain factor(s), other than an $A$ to $G$ transition at the nucleotide 3243 in the mitochondrial tRNA ${ }^{\text {LEU(UUR) }}$ gene (3243 mutation), may possibly distinguish between the pathogenesis and phenotypes for MELAS (mitochondriol encephalomyopathy, lactic acidosis and stroke-like episodes) and diabetes mellitus associated with this mutation.

They suggested with an analysis of diabetes and MELAS families which have appeared in recent reports, that this mutation may not be the only factor to define the clinical phenotypes of MELAS patients. The pathogenic mechanism underlying MELAS has been investigated intensively using various approaches. These studies have provided some insight into how the 3243 mutation results in MELAS. For example, any substitution at the strictly invariant nucleotide $A$ at position 3243 in the first base of the dihydrouridine loop of the tRNA molecule may disturb tRNA ${ }^{\text {LEU(UUR) }}$ function $[1,2]$ and under some specific conditions may affect the efficiency of transcription termination [3]. The studies using transformants obtained by transfer of mitochondria from MELAS patients into human mitochondrial DNA-less $(\varrho)$ cells showed

Corresponding author: Dr. M. Hashiramoto, Centre for Molecular and Cellular Biology, University of Queensland, St. Lucia, Brisbane, QLD 4072, Australia marked defects in mitochondrial protein synthesis and respiratory activity in transformants containing pure mutant mitochondrial DNA [4-6]. We feel that the number of pedigrees reviewed in the analysis by Drs. Odawara and Yamashita is not sufficient to contradict these achievements.

However, their argument does not lose its significance when we consider the facts that the 3243 mutation was reported to be found in only approximately $80 \%$ of the MELAS patients and that some patients carrying the 3243 mutation not only lacked MELAS symptoms but were not diabetic. These facts suggest that the clinical entity of the MELAS syndrome is in itself heterogeneous and cannot be explained by a single genetic abnormality. The fact that the severity of the oxidative phosphorylation defect and phenotype has been suggested to be attributed to aging also supports the requirement of the environmental factor for the development of MELAS.

In conclusion, we essentially agree with Drs. Odawara and Yamashita in that additional factor(s), other than the 3243 mutation, may be required to explain the variation of phenotype found in the patients with this mutation. However, we suggest that these factors, either genetic or environmental, are required to account for not only the MELAS symptoms but also the association of diabetes with this mutation. At present, we feel that we cannot neglect the possibility of diabetic patients carrying the 3243 mutation developing MELAS. Further investigation would be required to clarify this problem for the better understanding of these seemingly distinct disease entities.

Yours sincerely, M. Hashiramoto, M. Kishimoto, M. Kasuga 


\section{References}

1. Goto Y, Nonaka I, Horai S (1990) A mutation in the tRNA $^{\text {LEU(UUR) }}$ gene associated with the MELAS subgroup of mitochondrial encephalomyopathies. Nature 348: 651653

2. Tanaka M, Ino H, Ohbayashi T et al. (1991) Mitochondrial DNA mutations in mitochondrial myopathy, encephalopathy, lactic acidosis, and stroke-like episodes (MELAS). Biochem Biophys Res Commun 174: 861-868

3. Kruse B, Narashimhan N, Attardi G (1989) Termination of transcription in human mitochondria: identification of a DNA binding protein factor that promotes termination. Cell 58: 391-397

\section{Exclusion of adenosine deaminase gene locus on chromosome 20q12-13.1 in familial NIDDM in Taiwanese patients}

Dear Sir,

The linkage between adenosine deaminase gene locus on chromosome 20q12-13.1 and maturity-onset diabetes of the young (MODY, a subset of non-insulin-dependent diabetes mellitus [NIDDM] ) was found in the R-W pedigree $[1,2]$. Furthermore, defective beta-cell function has been demonstrated in the non-diabetic members of the R-W pedigree who had inherited the disease allele [3]. Those who inherited the disease allele at this locus may be predisposed to NIDDM; thus, this locus is a candidate gene locus for NIDDM. By parametric methods, adenosine deaminase locus was excluded as a cause of MODY in Edinburgh (UK), Wisconsin (USA) and French pedigrees [2, 4] and by non-parametric methods, no linkage was found between the adenosine deaminase locus and NIDDM in 50 diabetic sib sets from the United Kingdom and Italy [5]. However, its role in the pathogenesis of familial NIDDM in the Asian population has not been examined. We
4. Chomyn A, Martinuzzi A, Yoneda M et al. (1992) MELAS mutation in mtDNA binding site for transcription termination factor causes defects in protein synthesis and in respiration but no change in levels of upstream and downstream mature transcripts. Proc Natl Acad Sci USA 89: 4221-4225

5. Yoneda M, Chomyn A, Martinuzzi A, Hurko O, Attardi G (1992) Marked replicative advantage of human mtDNA carrying a point mutation that causes the MELAS encephalomyopathy. Proc Natl Acad Sci USA 89: 11164-11168

6. King MP, Koga Y, Davidson M, Schon EA (1992) Defects in mitochondrial protein synthesis and respiratory chain activity segregate with the tRNA ${ }^{\text {LEU(UUR) }}$ mutation associated with MELAS. Mol Cell Biol 12: 480-490 studied this locus in 213 subjects from 17 multiplex Taiwanese pedigrees with NIDDM with both LINKAGE (version 5.1) and affected-pedigree-member (APM) method (version 2.0) software programs.

In order to reduce the risk of missing a true linkage, linkage analysis was performed under four diagnostic schemes, based on the World Health Organization diagnostic criteria for diabetes with either high or low frequency of the disease gene. As shown in Figure 1, the linkage was rejected by the summed log of the odds (LOD) scores in most of the cases under an autosomal dominant model. Since NIDDM is a heterogenous disease, the A-test for heterogeneity among the studied pedigrees was tested based on the results of two-point linkage analyses by using the HOMOG (version 3.33) program of LINKAGE computer software package. No apparent heterogeneity was noted within the studied pedigree. Only one studied pedigree met the criteria for MODY (age at diagnosis ranging from 21 to 27 years) and linkage was also excluded in this pedigree.

Linkage analysis by the APM method provides another means to analyse a complex trait such as NIDDM without prior assumption of the mode of inheritance, since the mode of inheritance is unknown. However, the APM method is much more dependent on the allelic frequency; the allelic fre-

Table 1. Summary of summed log of the odds (LOD) scores for adenosine deaminase locus vs familial NIDDM

\begin{tabular}{|c|c|c|}
\hline Penetrance & Diagnostic scheme $^{\mathrm{a}}$ & Summed LOD score \\
\hline \multicolumn{3}{|c|}{ I: Frequency of disease gene: 0.0001} \\
\hline $100 \%$ & Overt diabetes, IGT vs unaffected & $-\infty$ \\
\hline Age-dependent ${ }^{\mathrm{a}}$ & Overt diabetes, IGT vs unaffected & -28.46 \\
\hline Age-dependent ${ }^{\mathrm{a}}$ & Overt diabetes, IGT vs unaffected & -20.52 \\
\hline- & Overt diabetes, IGT vs unaffected & -20.78 \\
\hline- & Overt diabetes vs unknown & -9.06 \\
\hline Age-dependent ${ }^{\mathrm{a}}$ & Overt diabetes, IGT vs unaffected & -7.47 \\
\hline $100 \%$ & Overt diabetes vs unaffected & -23.21 \\
\hline Age-dependent ${ }^{\mathrm{a}}$ & Overt diabetes, IGT vs unaffected & -6.46 \\
\hline- & Overt diabetes, IGT vs unaffected & -3.19 \\
\hline- & Overt diabetes vs unknown & -0.48 \\
\hline
\end{tabular}

age-dependent penetrance as described previously [2]

IGT, Impaired glucose tolerance

Corresponding author: Dr. B.J.Lin, Department of Internal Medicine and Graduate Institute of Clinical Medicine, National Taiwan University Hospital, Taipei, Taiwan 